\title{
Survey of diagnostic and typing capacity for Clostridium difficile infection in Europe, 2011 and 2014
}

SM van Dorp ${ }^{1}$, DW Notermans ${ }^{2}$, J Alblas ${ }^{2}$, P Gastmeier ${ }^{3}$, S Mentula 4 , E Nagy 5 , P Spigaglia 6 , K Ivanova ${ }^{7}$, F Fitzpatrick 8 , F Barbut ${ }^{9}$, T Morris ${ }^{10}$, MH Wilcox ${ }^{11}$, P Kinross ${ }^{12}$, C Suetens ${ }^{12}$, EJ Kuijper ${ }^{1}$, for the European Clostridium difficile Infection Surveillance Network (ECDIS-Net) project on behalf of all participants ${ }^{13}$

1. Department of Medical Microbiology, Leiden University Medical Centre, Leiden, the Netherlands

2. National Institute for Public Health and the Environment (RIVM), Bilthoven, the Netherlands

3. Institut für Hygiene und Umweltmedizin, Charité Universitätsmedizin Berlin, Berlin, Germany

4. National Institute for Health and Welfare (THL), Helsinki, Finland

5. Institute of Clinical Microbiology, University of Szeged, Szeged, Hungary

6. Istituto Superiore di Sanità, Rome, Italy

7. National Centre of Infectious and Parasitic Diseases, Sofia, Bulgaria

8. Health Protection Surveillance Centre and Beaumont Hospital, Dublin, Ireland

9. National Reference Laboratory for C. difficile, Paris, France

10. Public Health Wales, UK Anaerobe Reference Laboratory, Cardiff, United Kingdom

11. Leeds Teaching Hospitals NHS Trust, Leeds, United Kingdom

12. European Centre for Disease Prevention and Control, Stockholm, Sweden

13. Other members of the ECDIS-Net project are listed at the end of the article

Correspondence: Sofie M van Dorp (svandorp@lumc.nl)

Citation style for this article:

van Dorp SM, Notermans DW, Alblas J, Gastmeier P, Mentula S, Nagy E, Spigaglia P, Ivanova K, Fitzpatrick F, Barbut F, Morris T, Wilcox MH, Kinross P, Suetens C, Kuijper EJ, for the European Clostridium difficile Infection Surveillance Network (ECDIS-Net) project on behalf of all participants. Survey of diagnostic and typing capacity for Clostridium difficile infection in Europe, 2011 and 2014 . Euro Surveill. 2016;21(29):pii=30292. DOI: http://dx.doi.org/10.2807/1560-7917. ES.2016.21.29.30292

Suboptimal laboratory diagnostics for Clostridium difficile infection (CDI) impedes its surveillance and control across Europe. We evaluated changes in local laboratory CDI diagnostics and changes in national diagnostic and typing capacity for CDI during the European C. difficile Infection Surveillance Network (ECDIS-Net) project, through cross-sectional surveys in 33 European countries in 2011 and 2014. In 2011, $126(61 \%)$ of a convenience sample of 206 laboratories in 31 countries completed a survey on local diagnostics. In 2014, 84 (67\%) of these 126 laboratories in 26 countries completed a follow-up survey. Among laboratories that participated in both surveys, use of CDI diagnostics deemed 'optimal' or 'acceptable' increased from $19 \%$ to $46 \%$ and from $10 \%$ to $15 \%$, respectively $(p<0.001)$. The survey of national capacity was completed by national coordinators of 31 and 32 countries in 2011 and 2014, respectively. Capacity for any $C$. difficile typing method increased from 22/31 countries in 2011 to $26 / 32$ countries in 2014; for PCR ribotyping from 20/31 countries to $23 / 32$ countries, and specifically for capillary PCR ribotyping from 7/31 countries to $16 / 32$ countries. While our study indicates improved diagnostic capability and national capacity for capillary PCR ribotyping across European laboratories between 2011 and 2014, increased use of 'optimal' diagnostics should be promoted.

\section{Introduction}

Since 2003, Europe has been affected by outbreaks of Clostridium difficile infection (CDI) associated with the emergence of PCR ribotype 027/NAP1 [1]. A decade later, $C$. difficile was the microorganism responsible for $48 \%$ of healthcare-associated gastrointestinal infections in acute care hospitals across Europe [2]. Despite being frequent, $C D I$ remains underestimated in most European countries [3]. Underdiagnosis mainly results from a lack of awareness among medical doctors of when to suspect that patients may have CDI and use of suboptimal diagnostic algorithms at local microbiological laboratories [3-5]. Reference tests, i.e. toxigenic culture and cell culture cytotoxicity assay (CCA), are not suitable for routine application due to their complexity and long turnaround time [6,7]. Rapid enzyme immunoassays (EIAs) to detect $C$. difficile toxins in faeces lack sensitivity $[6,8]$. Highly sensitive tests such as EIA detecting glutamate dehydrogenase (GDH) - a $C$. difficile-specific enzyme [9] - or nucleic acid amplification tests (NAATs) have insufficient specificity $[6,10]$. To overcome underdiagnosis and suboptimal performance of stand-alone tests, the European Society of Clinical Microbiology and Infectious Diseases (ESCMID) has recommended since 2009 testing loose stools using two-step algorithms that have a highly sensitive test as the first screening step and a highly specific test as the second confirmatory test $[6,11]$. The 'Bristol stool scores' [12] are commonly used to categorise stool consistencies and can be used to select samples for 


\section{FIGURE 1}

Criteria for selection of faecal samples tested for Clostridium difficile among responding local laboratories that participated in the European Clostridium difficile Infection Surveillance Network (ECDIS-Net) project in $2011(\mathrm{n}=120)^{\mathrm{a}}$

Antibiotic-associated diarrhoea

Only on request of a medical doctor

Healthcare-associated diarrhoea

Loose stools

All faecal samples

\begin{tabular}{|cccc|c|}
\hline 1 & 1 & 30 & 40 & 50 \\
0 & 10 & 20 & 30 & 40
\end{tabular}

Percentage of laboratories

a Laboratories in 31 countries responded to the 2011 survey: Austria, Belgium, Bulgaria, Croatia, Cyprus, Czech Republic, Denmark, Estonia, Finland, France, Germany, Greece, Hungary, Iceland, Ireland, Italy, Latvia, Lichtenstein, Lithuania, Luxembourg, the Netherlands, Norway, Poland, Portugal, Romania, Slovenia, Spain, Sweden, Switzerland, Turkey and the United Kingdom (not including Wales). Serbia did not participate in the European Clostridium difficile Infection Surveillance Network (ECDIS-Net) project in 2011. No laboratories in Slovakia and Wales were invited to participate by ECDIS-Net national coordinators in 2011.

\section{FIGURE 2}

Reported changes affecting national/subnational laboratory diagnostic capacity for Clostridium difficile infection between 2011 and 2014 in participating European countries $(n=32)^{\mathrm{a}}$

Availability of tests on the market

New/revised national guidelines

No change

Other

Change in legislation

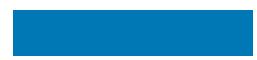

Change in reimbursement of diagnostic tests

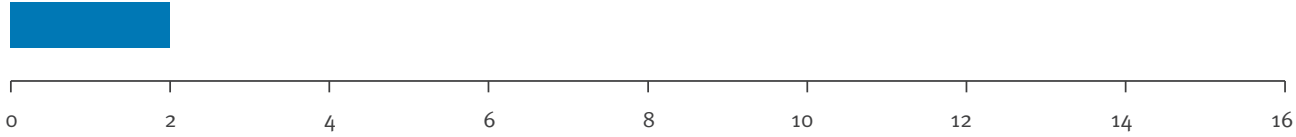

Number of countries

a Austria, Belgium, Bulgaria, Croatia, Cyprus, Czech Republic, Denmark, Estonia, Finland, France, Germany, Greece, Hungary, Ireland, Italy, Latvia, Lichtenstein, Lithuania, Luxembourg, the Netherlands, Norway, Poland, Portugal, Romania, Serbia, Slovakia, Slovenia, Spain, Sweden, Switzerland, Turkey and the United Kingdom (data were analysed separately for England, Northern Ireland, Scotland and Wales, but counted as one country). No data were available for Iceland.

b Seven countries reported other changes in national laboratory diagnostics: Slovenia was developing new national guidelines for CDI at the time of the second survey; Romania started a national surveillance study in 2014; Spain published an opinion document on CDI [32]; Slovakia was in the process of implementing new diagnostic methods due to an increased interest in CDI; in Cyprus, the central diagnostic laboratory for C. difficile implemented a two-step diagnostic algorithm; in Finland, CDI diagnostics were subcontracted to laboratory consortia that applied nucleic acid amplification tests more often; and Hungary relocated its national reference laboratory to expand its laboratory capacity but still had limited resources. 


\section{FIGURE 3}

Clostridium difficile typing methods available in countries that participated in the European Clostridium difficile Infection Surveillance Network (ECDIS-Net) project in $2011(\mathrm{n}=31)^{\mathrm{a}}$ and $2014(\mathrm{n}=32)^{\mathrm{a}}$
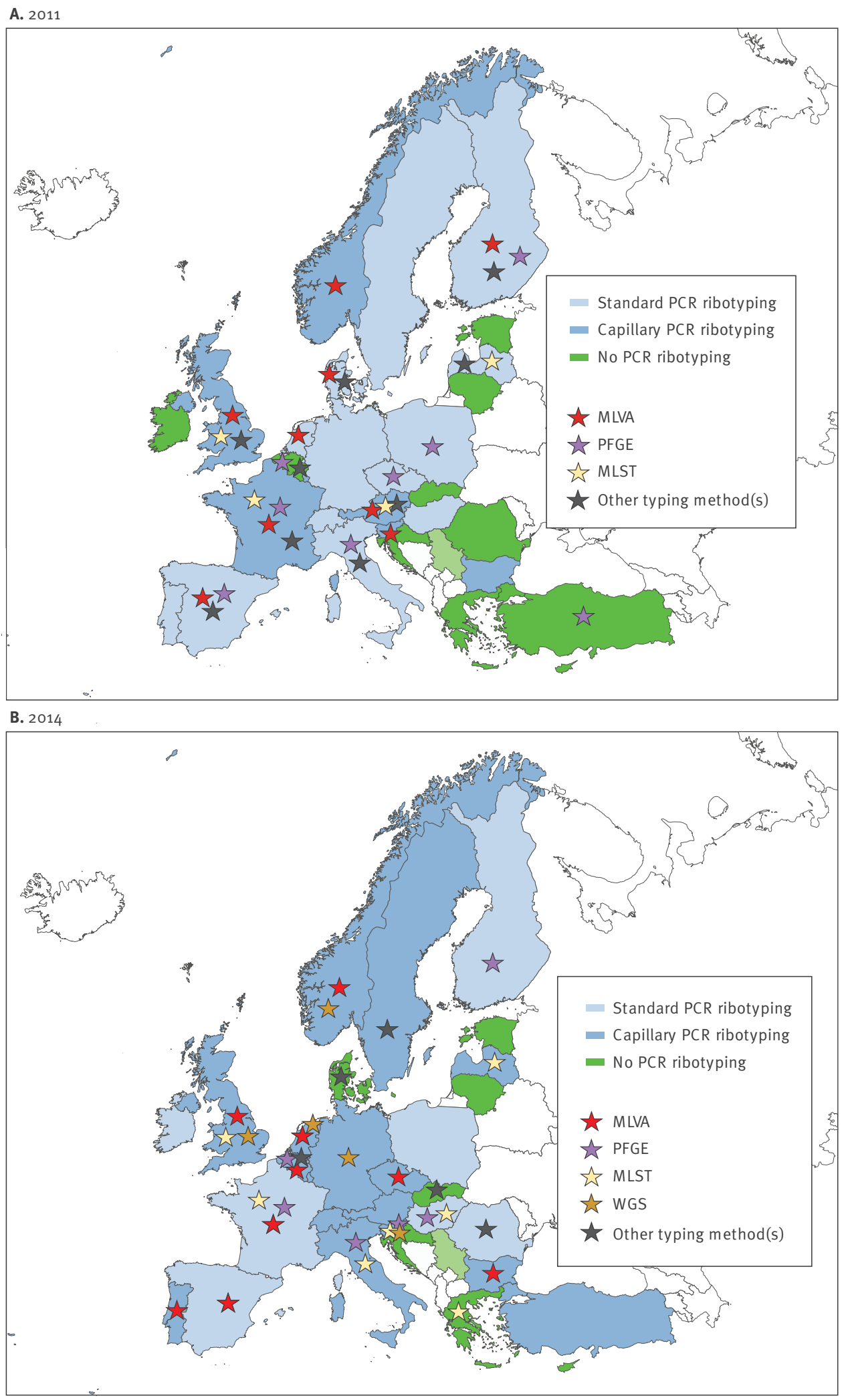

PFGE: pulsed-field gel electrophoresis; MLST: multilocus sequence typing; MLVA: multilocus variable-number tandem repeat analysis; WGS: whole genome sequencing. Other typing methods used in 2011 were: tcdC typing (Austria, Belgium, Finland, France, Italy, Latvia, Luxembourg (not shown), Spain, United Kingdom - Northern Ireland only), repetitive-element PCR (Belgium, Spain), toxinotyping (Italy, Spain), tandem repeat sequence typing (Denmark) and pathogenicity locus (PaLoc) multiplex PCR (Finland).

Other typing methods used in 2014 were: tcdA/B (Belgium, Romania, Slovakia), CDT (Belgium, Slovakia), tcdC (Belgium), $\Delta 117$ TcdC (Slovakia), and GyrA $\Delta$ detection (Belgium) detection, tandem repeat sequence typing (Denmark), and high molecular weight typing by MALDI-TOF (Sweden).

a In 2011, 31 countries responded: Austria, Belgium, Bulgaria, Croatia, Cyprus, Czech Republic, Denmark, Estonia, Finland, France, Germany, Greece, Hungary, Ireland, Italy, Latvia, Lichtenstein, Lithuania, Luxembourg, the Netherlands, Norway, Poland, Portugal, Romania, Slovakia, Slovenia, Spain, Sweden, Switzerland, Turkey and the United Kingdom (not including Wales). No data were available for Iceland. In 2011, Serbia did not participate in the European Clostridium difficile Infection Surveillance Network (ECDIS-Net) project. In 2014, Serbia participated in the ECDIS-Net project and responded to the 2014 questionnaire, as did Wales, and so the number of responding countries in 2014 was 32.

Source of map: FreeVectorMaps.com (http://freevectormaps.com). 
Criteria for categorisation of Clostridium difficile infection diagnostic algorithms, survey of European countries participating in the European Clostridium difficile Infection Surveillance Network (ECDIS-Net) project, 2011 ( $\mathrm{n}=31)^{\mathrm{a}}$ and $2014(\mathrm{n}=26)^{\mathrm{a}}$

\begin{tabular}{|c|c|c|c|}
\hline \multirow{2}{*}{\multicolumn{2}{|c|}{$\begin{array}{l}\text { Categorisation of CDI diagnostics } \\
\text { Screening test }\end{array}$}} & \multicolumn{2}{|c|}{ CDI diagnostic algorithm } \\
\hline & & Confirmatory test & \\
\hline \multirow{2}{*}{ Optimal ${ }^{b}$} & $1^{\mathrm{c}}$ & NAAT & EIA toxin detection \\
\hline & $2-3^{c}$ & GDH EIA and toxin detection & NAAT or toxigenic culture \\
\hline \multirow{2}{*}{ Acceptable $^{b}$} & $4-5^{c}$ & GDH EIA detection & NAAT or toxigenic culture \\
\hline & $6^{c}$ & NAAT & None \\
\hline Incomplete $^{\mathrm{b}}$ & $7-10^{c}$ & \multicolumn{2}{|c|}{ All other algorithms } \\
\hline
\end{tabular}

CDI: Clostridium difficile infection; EIA: enzyme immunoassay; GDH: glutamate dehydrogenase; NAAT: nucleic acid amplification test.

a Laboratories in 31 countries responded to the 2011 survey: Austria, Belgium, Bulgaria, Croatia, Cyprus, Czech Republic, Denmark, Estonia, Finland, France, Germany, Greece, Hungary, Iceland, Ireland, Italy, Latvia, Lichtenstein, Lithuania, Luxembourg, the Netherlands, Norway, Poland, Portugal, Romania, Slovenia, Spain, Sweden, Switzerland, Turkey and the United Kingdom (not including Wales). Serbia did not participate in the European Clostridium difficile Infection Surveillance Network (ECDIS-Net) project in 2011. No laboratories in Slovakia and Wales were invited to participate by ECDIS-Net national coordinators in 2011. Laboratories in 26 countries responded in 2014 (no data from laboratories in Croatia, Iceland, Latvia, Slovenia and Switzerland).

${ }^{b}$ Categorisation of CDI diagnostic algorithms in the second survey, in 2014 [21].

c Corresponding CDI diagnostic algorithms in the second survey, in 2014 [21].

CDI testing. ESCMID recommended performing CDI testing not only upon request of a medical doctor, but also based on other indications such as the 'three-day rule', i.e. diarrhoea after three days of hospitalisation or when diarrhoea develops after antibiotic use $[6,13]$.

The type of diagnostic algorithm applied influences not only clinical care [14], but also CDI surveillance's sensitivity and specificity $[3,14,15]$. However, a consensus on when and how to test for CDI has not been established among reference and local laboratories.

Additionally, typing of $C$. difficile to understand its local or wider transmission remains non-standardised in Europe $[16,17]$. Numerous typing methods have become available for routine use in the last 30 years. For $C$. difficile, these include methods that use restriction enzymes (e.g. restriction endonuclease analysis, pulsed-field gel electrophoresis (PFGE)), PCR amplification of housekeeping genes (e.g. multilocus sequence typing (MLST)), of repetitive elements (repetitive-element $P C R$, multilocus variable-number tandem repeat analysis (MLVA)), of the pathogenicity locus (e.g. toxinotyping) or of $16 \mathrm{~S}-23 \mathrm{~S}$ rRNA intergenic spacer regions (e.g. PCR ribotyping) $[16,18]$. Whole genome sequencing, with its ultimate discriminatory power, can already be used for in-depth analysis of evolutionary patterns [19]. Nevertheless, PCR ribotyping still remains the standard typing method in Europe as it involves relatively simple technology and its low costs permits widespread application $[16,18]$.

In 2010, the European Centre for Disease Prevention and Control (ECDC) launched the European C. difficile Infection Surveillance Network (ECDIS-Net) project, an initiative to enhance and harmonise laboratory diagnostic and typing capacity for CDI, and to support surveillance of CDI in Europe. The project consortium consisted of a team of experts involved in the first
European multicountry surveillance study performed in 2008 [20]. Between 2010 and 2014, the ECDIS-Net project developed standard operating procedures for C. difficile culturing and PCR ribotyping, implemented a reference nomenclature database and compiled a set of reference strains to standardise PCR ribotyping. National reference laboratories were invited to participate in a workshop for culturing and typing of $C$. difficile and participated in an external quality assessment exercise.

The study presented here measured changes in capacity for diagnostic testing for CDI and typing of $C$. difficile isolates in Europe between 2011 and 2014, using surveys of European local laboratories and national coordinators participating in the ECDIS-Net project. Additionally, we aimed to obtain insight into barriers to optimal CDI laboratory diagnostics, to inform further activities of ECDC and of the ESCMID Study Group for C. difficile (ESGCD) in this field.

\section{Methods}

\section{Study design}

The Dutch National Reference Laboratory for C. difficile (Leiden University Medical Centre, Leiden, and the National Institute for Public Health and the Environment (RIVM), Bilthoven, the Netherlands) coordinated data collection in 2011 and in 2014 by cross-sectional surveys among two target groups: (i) local microbiology laboratories, in order to evaluate changes in routine laboratory diagnostics; and (ii) national coordinators, i.e. representatives of national or regional reference laboratories nominated by competent bodies for surveillance on the request of ECDC, to evaluate national changes in diagnostic and typing capacity for $C$. difficile. In 2011 and 2014, 32 and 33 countries participating in the ECDIS-Net project were invited to take part in 
the survey, respectively (in 2011, Serbia did not participate in ECDIS-Net). All surveys are available online [21].

\section{Selection}

There was no European register of microbiology laboratories to use for random sampling. Therefore, ECDISNet national coordinators were requested to invite a representative sample of the local clinical microbiology laboratories (about 10\%) in each country to participate in the survey. In Austria and Norway, the laboratories were selected by random sampling; all other countries used non-random convenience sampling [22]. Selected laboratories were emailed an initial survey in October 2011: some laboratories replied in 2012. All respondents to the initial survey received a follow-up survey in June 2014.

\section{Data collection}

Data were collected through a centralised web-based system (Questback, New York, United States). In 2011, the initial survey contained questions on several aspects of local routine diagnostics, including indications for undertaking CDI diagnostics and methodologies. Laboratories were requested to report the type of screening test primarily used for CDI diagnostics and confirmatory test (if applicable). For both, they could report more than one test. In 2014, the follow-up survey listed 10 diagnostic algorithms each designated as either 'optimal', 'acceptable' or 'incomplete' (Table 1). Laboratories were requested to estimate the percentage of samples that had been tested according to each algorithm listed, or to describe their usual diagnostic algorithm and estimate the corresponding percentage. The categorisation of CDI diagnostic algorithms was made by some of the ECDIS-Net experts who were also involved in revising the ESCMID diagnostics guidelines for CDI [6]. Algorithms designated as optimal had high sensitivity and specificity (not specifically defined), detection of free toxins in faeces and a rapid turnaround time [23]. Acceptable algorithms met the same criteria but without detecting free toxins in faeces. Any other algorithm was designated as incomplete. The 2014 follow-up survey additionally contained questions on barriers to apply optimal or acceptable diagnostic algorithms and changes in the indications for sending samples for CDI diagnosis by medical doctors.

\section{Data analysis}

To allow comparison, data on diagnostics from the 2011 initial survey were distributed into the three categories of diagnostic algorithms defined in 2014. For each local laboratory, CDI diagnostics, i.e. CDI testing practices, were considered optimal if more than $80 \%$ of the samples followed an optimal diagnostic algorithm, and acceptable ifmore than $80 \%$ of the samples followed either an optimal or acceptable algorithm. CDI diagnostics of all other algorithms were considered incomplete. When a laboratory reported a three-step algorithm by applying a third diagnostic test when the screening and confirmatory tests were contradictory, this algorithm was allocated to the best-matching twostep algorithm. Changes in local laboratory diagnostic capacity were evaluated by the McNemar's test [24], and changes in the use of optimal, acceptable and incomplete algorithms in 2011 and 2014 were evaluated by a Bowker test for symmetry [24]. A sensitivity analysis was performed using two assumptions on missing data in 2014, i.e. CDI diagnostics one category inferior (Table 1) than in 2011 and CDI diagnostics one category superior than in 2011. Data were analysed using IBM SPSS statistics 20 (SPSS Inc., Chicago, United States).

\section{Survey of ECDIS-Net national coordinators}

Data collection and analysis

All ECDIS-Net national coordinators received an initial survey in May 2011 and a follow-up survey in June 2014. Both surveys contained questions on national typing capacity (defined as any laboratory in the country performing typing) and on molecular typing methods, asking which were available in their country from a list of common methods [18].

\section{Results}

\section{Local laboratory capacity}

\section{Participants}

Questionnaires on local diagnostic and typing capacity for CDI were completed by 126 (61\%) of 206 laboratories in 2011-12 and by 84 (67\%) of these same 126 laboratories in 2014 (Table 2). A total 124 (98\%) of the 126 responding laboratories in 2011-12 provided microbiological services to hospitals, of which 103 (83\%) served at least one university, secondary or tertiary care hospital. In addition, 66 (53\%) provided microbiological services to long-term care facilities, of which 45 provided services to nursing homes. Furthermore, 65/124 ( $52 \%$; data were missing for two laboratories) provided medical services to other healthcare services (e.g. general practitioners). In 2011 and 2014, 120/126 (95\%) and $83 / 84$ laboratories (99\%, among responders to both questionnaires; $p=0.50$ ), respectively, reported that they performed CDI laboratory diagnostics.

Indications for Clostridium difficile infection diagnostics The indications for CDI diagnostics reported in 2011 are listed in Figure 1. In 2014, a change of indications for sending samples for CDI diagnosis by medical doctors was observed; 16 (19\%) of 83 laboratories reported that one or two changes had occurred since 2011. Several laboratories introduced the use of Bristol stool scores to assess stool consistency for sample selection $(n=5)$. Also, patient populations that were previously not monitored for CDI (e.g. outpatients, high-risk populations) were later explicitly included in protocols $(n=3)$ and awareness and recognition of CDI among clinicians had improved $(n=5)$. Other improvements of sample selection were also reported $(n=5)$, i.e. application of guidelines for sample selection $(n=3)$ and/ or the three-day rule, i.e. diarrhoea after three days 
TABLE 2

Response of participating European countries to local laboratory ( $\mathrm{n}=31$ and $\mathrm{n}=26$, respectively) and national/subnational surveys ( $\mathrm{n}=31$ and $\mathrm{n}=32$, respectively) on Clostridium difficile infection diagnostic and typing capacity, 2011 and 2014

\begin{tabular}{|c|c|c|c|c|}
\hline \multirow[t]{2}{*}{ Country } & \multicolumn{2}{|c|}{$\begin{array}{c}\text { Number of laboratories that responded to local questionnaire / } \\
\text { number invited }\end{array}$} & \multicolumn{2}{|c|}{ Replied to national questionnaire } \\
\hline & 2011 & 2014 & 2011 & 2014 \\
\hline Austria & $4 / 8$ & $2 / 4$ & Yes & Yes \\
\hline Belgium & $4 / 9$ & $4 / 4$ & Yes & Yes \\
\hline Bulgaria & $7 / 7$ & $2 / 7$ & Yes & Yes \\
\hline Croatia & $2 / 4$ & $0 / 2$ & Yes & Yes \\
\hline Cyprus & $3 / 3$ & $3 / 3$ & Yes & Yes \\
\hline Czech Republic & $9 / 11$ & $7 / 9$ & Yes & Yes \\
\hline Denmark & $3 / 3$ & $1 / 3$ & Yes & Yes \\
\hline Estonia & $2 / 2$ & $1 / 2$ & Yes & Yes \\
\hline Finland & $3 / 3$ & $2 / 3$ & Yes & Yes \\
\hline France & $5 / 37$ & $2 / 5$ & Yes & Yes \\
\hline Germany & $5 / 7$ & $5 / 5$ & Yes & Yes \\
\hline Greece & $3 / 3$ & $2 / 3$ & Yes & Yes \\
\hline Hungary & $8 / 8$ & $8 / 8$ & Yes & Yes \\
\hline Iceland & $1 / 1$ & $0 / 1$ & No & No \\
\hline Ireland & $3 / 5$ & $2 / 3$ & Yes & Yes \\
\hline Italy & $13 / 14$ & $8 / 13$ & Yes & Yes \\
\hline Latvia & $2 / 3$ & $0 / 2$ & Yes & Yes \\
\hline Lichtenstein & $1 / 1$ & $1 / 1$ & Yes & Yes \\
\hline Lithuania & $3 / 3$ & $2 / 3$ & Yes & Yes \\
\hline Luxembourg & $2 / 6$ & $1 / 2$ & Yes & Yes \\
\hline Netherlands & $4 / 6$ & $3 / 4$ & Yes & Yes \\
\hline Norway & $9 / 13$ & $4 / 9$ & Yes & Yes \\
\hline Poland & $6 / 6$ & $4 / 6$ & Yes & Yes \\
\hline Portugal & $4 / 5$ & $4 / 4$ & Yes & Yes \\
\hline Romania & $4 / 6$ & $3 / 4$ & Yes & Yes \\
\hline Serbiab $^{b}$ & NA & NA & NA & Yes \\
\hline Slovakiac & NA & NA & Yes & Yes \\
\hline Slovenia & $1 / 3$ & $0 / 1$ & Yes & Yes \\
\hline Spain & $3 / 5$ & $2 / 3$ & Yes & Yes \\
\hline Sweden & $2 / 3$ & $2 / 2$ & Yes & Yes \\
\hline Switzerland & $1 / 1$ & $0 / 1$ & Yes & Yes \\
\hline Turkey & $2 / 7$ & $2 / 2$ & Yes & Yes \\
\hline UK-England & $2 / 6$ & $2 / 2$ & Yes & Yes \\
\hline UK-Northern Ireland & $1 / 3$ & $1 / 1$ & Yes & Yes \\
\hline UK-Scotland & $4 / 4$ & $4 / 4$ & Yes & Yes \\
\hline UK-Wales ${ }^{c}$ & NA & NA & No & Yes \\
\hline Total & $126 / 206$ & $84 / 126$ & 31 & 32 \\
\hline
\end{tabular}

NA: not applicable; UK: United Kingdom.

${ }^{a}$ For the UK, data were analysed separately for England, Northern Ireland, Scotland and Wales, but the UK was counted as one country.

b Serbia did not participate in the European Clostridium difficile Infection Surveillance Network (ECDIS-Net) project in 2011.

c No laboratories in Slovakia and Wales were invited to participate by ECDIS-Net national coordinators.

of hospitalisation $(n=1)$, and unspecified attempts to improve sample selection $(n=1)$.

C. difficile infection diagnostics

In 2011,17 (14\%) of 120 laboratories had optimal CDI diagnostics, 12 (10\%) acceptable diagnostics and 91 (76\%) incomplete diagnostics (Table 3 ). Incomplete algorithms included use of EIA toxin detection for screening with or without a confirmatory test, or a combination of EIA GDH and toxin detection without other tests for confirmation. Among laboratories responding to both the 2011 and 2014 surveys and that performed CDI diagnostics at both time-points $(n=81)$, the percentage of laboratories with optimal CDI diagnostics 
increased from $19 \%$ to $46 \%$ and that with acceptable CDI diagnostics from $10 \%$ to $15 \%$ while the percentage of laboratories with incomplete CDI diagnostics decreased from $72 \%$ to $40 \%$ ( $p<0.001$; Table 3 ). Two laboratories without any diagnostics in 2011 had optimal and incomplete CDI diagnostics, respectively, in 2014 .

\section{Sensitivity analysis}

Laboratories with optimal CDI diagnostics in 2011 were more likely to respond to the 2014 survey (15/17) compared with those with acceptable $(8 / 12)$ or incomplete diagnostics (58/91). Under the negative assumption that all non-responding laboratories in 2014 applied CDI diagnostics one category inferior in 2014 compared with that of 2011, the percentage of laboratories with optimal diagnostics would have increased from $14 \%$ to $31 \%$, that with acceptable diagnostics would have increased from $10 \%$ to $12 \%$, and that with incomplete diagnostics would have decreased from $76 \%$ to $58 \%$ between 2011 and 2014 ( $p<0.001$ ). Conversely, if all non-responding laboratories had CDI diagnostics one category superior in 2014 compared with 2011 , the percentage of laboratories with optimal diagnostics would have increased from $14 \%$ to $36 \%$, that with acceptable diagnostics would have increased from 10 to $38 \%$, and that with incomplete diagnostics would have decreased from 76 to $27 \%$ between 2011 and 2014 ( $p<0.001$ ).

Barriers to optimal/acceptable diagnostics for C. difficile infection

Barriers to applying optimal or acceptable algorithms were examined in 2014. Of the 33 laboratories with incomplete CDI diagnostics, 17 indicated that materials or tests were too costly, six indicated receiving insufficient reimbursement for tests from insurers and five had insufficient availability of trained staff. Of the 50 laboratories that had optimal or acceptable CDI diagnostics, 10 also indicated that materials or tests were too costly, seven indicated receiving insufficient reimbursement from insurers and five had insufficient availability of trained staff. Ten laboratories that responded in 2014 indicated that they disagreed with the project's designations of the CDI diagnostic algorithms as optimal, acceptable or incomplete.

\section{National/subnational capacity}

\section{Participating countries}

The national coordinators of 31 and 32 countries responded to the national survey in 2011 and 2014, respectively (Table 2 ). Data were collected separately for the four countries within the United Kingdom (UK), i.e. England, Northern Ireland, Scotland and Wales, but the UK was counted as one country.

\section{Changes in national diagnostic capacity}

In 2014, eight of the 32 responding countries (France, Germany, Greece, Latvia, Luxembourg, Sweden, Switzerland, Turkey) reported no change in national/ subnational laboratory diagnostics for CDI. Conversely,
24 countries reported one or more changes in national/ subnational laboratory diagnostics for CDI since 2011 (Figure 2). Specifically, 16 countries had experienced a change in availability of commercial diagnostic tests (Bulgaria, Czech Republic, Estonia, Hungary, Ireland, Italy, Lichtenstein, Lithuania, the Netherlands, Norway, Poland, Portugal, Romania, Serbia, Slovenia, UK), 10 countries had new or revised guidelines for CDI diagnostics (Austria, Czech Republic, Denmark, Estonia, Hungary, Ireland [25], Italy, Lithuania, Romania, UK) and three countries had changes in relevant legislation (Hungary, Poland, Romania). Three countries (Belgium, Croatia, Czech Republic) had implemented changes in reimbursement policies for diagnostic tests. Greece had limited access to and reimbursement of materials in both 2011 and 2014. In 2012, the UK implemented 'harmonised' diagnostics using GDH screening (or NAAT) and EIA toxin detection (or CCA) in all its laboratories [26].

\section{C. difficile national typing methods}

The capacity for various $C$. difficile typing methods in participating countries in 2011 and 2014 is depicted in Figure 3. The number of countries able to perform any method of typing increased from 22/31 countries in 2011 to $26 / 32$ countries in 2014. Only six countries (Croatia, Cyprus, Estonia, Lichtenstein, Lithuania, Serbia) reported that they did not have any national typing capacity in 2014 (none of these countries had typing capacity in 2011); however, Lichtenstein sent samples to another country (Austria) for typing.

Several typing methods were implemented by the countries (Figure 3). PCR ribotyping (either capillarybased or conventional agarose gel-based), the current European standard for $C$. difficile typing, was available in 20/31 countries in 2011 and in 23/32 countries in 2014. Two of the countries that acquired ribotyping capacity (Ireland and Romania) use it for national surveillance. Capillary PCR ribotyping was applied by 7/31 countries in 2011 and by 16/32 countries in 2014 . In 2014, nine of the 32 participating countries applied MLVA, six PFGE and seven MLST. In 2014, whole genome sequencing was available in Germany, the Netherlands, Norway, Slovenia and England.

Some countries reported specific changes in national molecular typing capacity between 2011 and 2014 . Greece, which previously did not have typing capacity, introduced MLST in January 2014. At the time of the 2014 survey, Estonia was capable of ribotyping for research projects, although there were no such projects. Turkey performed PCR ribotyping but lacked software to analyse the data. Denmark stopped using PCR ribotyping and only applied tandem repeat sequence typing. Hungary reported limited typing capacity for financial reasons although PCR ribotyping remained available at the national reference laboratory. Finland restricted the indications for ribotyping to severe CDI or outbreaks, which unintentionally caused many 
Laboratories participating in the European Clostridium difficile Infection Surveillance Network (ECDIS-Net) project according to their diagnostics category, $2011(\mathrm{n}=120)$ a and $2014(\mathrm{n}=81)^{\mathrm{a}}$

\begin{tabular}{|c|c|c|c|}
\hline \multirow{3}{*}{ Categorisation of CDI diagnostics ${ }^{b}$} & \multirow{2}{*}{$\begin{array}{l}\text { All laboratories that provided } \\
\text { data } \\
2011\end{array}$} & \multicolumn{2}{|c|}{$\begin{array}{l}\text { Only laboratories that provided data in both } 2011 \text { and } \\
\qquad 2014 \text { surveys }\end{array}$} \\
\hline & & 2011 & $2014^{c}$ \\
\hline & n (\%) & $n(\%)^{d}$ & $n(\%)^{d}$ \\
\hline Optimal & $17(14)$ & $15(19)$ & $37(46)$ \\
\hline Acceptable & $12(10)$ & $8(10)$ & $12(15)$ \\
\hline Incomplete & $91(76)$ & $58(72)$ & $32(40)$ \\
\hline Total & $120(100)$ & $81(100)$ & $81(100)$ \\
\hline
\end{tabular}

CDI: Clostridium difficile infection.

${ }^{a}$ Laboratories in 31 countries responded to the 2011 survey: Austria, Belgium, Bulgaria, Croatia, Cyprus, Czech Republic, Denmark, Estonia, Finland, France, Germany, Greece, Hungary, Iceland, Ireland, Italy, Latvia, Lichtenstein, Lithuania, Luxembourg, the Netherlands, Norway, Poland, Portugal, Romania, Slovenia, Spain, Sweden, Switzerland, Turkey and the United Kingdom (not including Wales). Serbia did not participate in the European Clostridium difficile Infection Surveillance Network (ECDIS-Net) project in 2011. No laboratories in Slovakia and Wales were invited to participate by ECDIS-Net national coordinators in 2011. Laboratories in 26 countries responded in 2014 (no data from laboratories in Croatia, Iceland, Latvia, Slovenia and Switzerland).

${ }^{b}$ CDI diagnostics were considered 'optimal' if $>80 \%$ of the samples followed an 'optimal' testing algorithm, and 'acceptable' if $>80 \%$ of the samples followed either an 'optimal' or 'acceptable' testing algorithm. CDI diagnostics of all other laboratories were considered 'incomplete'. The diagnostic algorithms are described in Table 1.

c Two laboratories that did not perform CDI laboratory diagnostics in 2011 were not included. These laboratories indicated in the 2014 questionnaire that they used optimal and incomplete CDI diagnostics, respectively.

${ }^{d}$ The percentages in this column do not add up to 100 due to rounding.

laboratories to stop all culturing and/or sending isolates for typing.

\section{Discussion}

This study assessed changes in diagnostic testing and typing capacity for CDI in Europe between 2011 and 2014, using surveys of European local laboratories and of national coordinators participating in the ECDIS-Net project. Virtually all participating local laboratories had implemented CDI diagnostics in 2011 and 2014 , compared with $88 \%(186 / 212)$ of the local laboratories investigated in eight European countries in 2003 [27]. The percentage of laboratories with optimal CDI diagnostics increased from $19 \%$ to $46 \%$, and that with acceptable diagnostics increased from $10 \%$ to $15 \%$. Importantly, the ESCMID-recommended twostep diagnostic algorithm [6] became more common. Nevertheless, we still observed a considerable variation in CDI diagnostics within and between European countries, in line with another European study with 482 participating hospitals in 2011-13 [3]. This variation in diagnostics can substantially affect CDI incidence rates obtained by surveillance $[15,28]$. Our survey showed that suboptimal CDI diagnostics may result from, for example, financial restrictions or limited availability of trained staff. As a consequence of the disagreement by a sizable minority of laboratories with the designation of diagnostic algorithms, the ESGCD undertook to revise its diagnostic guidelines [6] and propose an algorithm that can also be implemented in laboratories with limited numbers of trained staff and limited financial resources. These revised guidelines will be published in 2016 on behalf of ESCMID.

Among countries having national guidelines available, the UK was the only one that had succeeded in harmonising CDI diagnostics, by recommending a single two-test diagnostic algorithm ('comprising a GDH EIA (or NAAT/PCR) followed by a sensitive toxin EIA') $[3,26]$. The recommendations in the UK Department of Health guidance were supported by local study data and inclusion of frequently asked questions to allay objections of the laboratories to implementing the proposed diagnostic algorithms [26]. Furthermore, the diagnostics guidance was one of many $C$. difficilerelated activities in the UK, for example, implementation of mandatory CDI reduction targets with financial penalties for national health services [29]. There probably are two possible ways to optimise testing: either to promote one national diagnostic algorithm or to promote the use of optimal testing strategies by local laboratories. However, the proposed algorithm in the UK was not fully compliant with the designation of diagnostic algorithms as optimal in this survey, highlighting the need for further discussion among experts to reach a consensus. Another example is Spain, where several national studies and meetings were organised $[30,31]$ that resulted in an opinion document to enhance optimal diagnostics for CDI [32]. We hope that the national reference laboratories that participated in the ECDIS-Net project will follow these examples and promote optimal diagnostics for CDI and its implementation in local laboratories.

\section{Typing capacity}

Between 2011 and 2014, PCR ribotyping capacity and capillary PCR ribotyping increased among the participating countries. Capillary PCR ribotyping was validated in 2012-14 by four reference laboratories in England, the Netherlands, the United States and Canada, identifying a $98 \%$ consensus (195/200 cases tested) between the laboratories, which indicated the 
method's suitability for standardised CDI surveillance [17].

We assume that ECDIS-Net activities during 2012-14, including a training programme for $C$. difficile PCR ribotyping, contributed to the increased $P C R$ ribotyping capacity. For example, Romania joined the training programme in $\mathbf{2 0 1 2}$ and received a set of reference strains from the ECDIS-Net project and is now able to apply $P C R$ ribotyping in their national surveillance. Poland reported having started their first national surveillance programme, stimulated by ECDIS-Net activities in 2012 [33]. A few countries (Hungary, Italy, Slovenia) had national surveillance under development at time of the 2014 survey. Despite these positive trends, our study also indicates that some European reference and local laboratories are affected by limited resources and budget reductions, which hamper implementation and technical improvements of molecular typing methods.

\section{Limitations}

This study has several limitations including the small, non-random selection of local laboratories for both surveys and the moderate response rate, limiting the degree to which conclusions can be extrapolated to all European microbiological laboratories. The representativeness of the invited and participating laboratories could not be assessed due to the absence of a suitably complete European register. Laboratories with better CDI diagnostics may have been more likely to participate in the original and follow-up surveys, leading to an overestimation of the number of laboratories with optimal CDI diagnostics in Europe. Additionally, the categorisation of CDI diagnostic algorithms into three levels, although made through a series of consultations with a team of international experts from the ECDIS-Net project, was based on expert opinion and some subjectivity cannot be excluded. Also, although the 2014 questionnaire for local laboratories requested quantitative data on the percentage of tests that followed each algorithm on a provided list, as the list had the subheadings 'optimal', 'acceptable' and 'incomplete', it is possible that those responding overestimated the proportion of desirable answers. We estimate that this reporting bias was minimal as for almost all laboratories, just one algorithm was used.

\section{Conclusions}

We conclude that the ECDIS-Net project laid the foundations for Europe-wide surveillance of CDI, although increased use of optimal diagnostic algorithms should be promoted, taking into consideration the limited resources and budget cuts in several European countries. The ESGCD revised the ESCMID diagnostics guidelines for CDI, which, once published, should contribute to standardisation of CDI diagnostics at local and national level in Europe. Typing capacity for CDI in Europe was acceptable overall; however, an internationally standardised capillary PCR ribotyping protocol is now available [17] and requires further implementation in European countries. We would recommend that these important steps are considered as part of the integration of $C$. difficile molecular typing data in The European Surveillance System (TESSy), within the ECDC-coordinated Europe-wide CDI surveillance (since 1 January 2016) [34].

\section{Other members of the ECDIS-Net project}

F Allerberger, D Schmid (AGES, Austria); M Delmée, J van Broeck, (University of Louvain, Belgium); R VatchevaDobrevska (National Centre of Infectious and Parasitic Diseases, Bulgaria); B Matica (Dr. Andrija Štampar Insitute of Public Health, Croatia); P Maikanti-Charalampous, D Pieridou- Bagatzouni (Nicosia General Hospital, Cyprus); 0 Nyč (Hospital FN Motol Prague, Czech Republic); KEP Olsen (Statens Serum Institut, Denmark); M Jyrna-Ellam, Anastasia Pavelkovich (North-Estonian Regional Hospital, Estonia); A Virolainen-Julkunen, O Lyytikäinen, (Institute of Health and Welfare, Finland); C Eckert (National Reference Laboratory for C. difficile, France); B Coignard (Institut de Veille Sanitaire, France); L von Müller (University of Saarland Medical Centre, Germany); B Weiß (Robert Koch-Institut, Germany); E Petinaki (University Hospital of Larissa, Greece), A Kurcz, Z Barna, A Hajdu (National Center for Epidemiology, Hungary); L Kyne, L Fenelon (Health Protection Surveillance Centre and Beaumont Hospital, Ireland); F Barbanti (Istituto Superiore di Sanità, Italy), ML Moro (Agenzia Sanitaria e Sociale Regionale Emilia-Romagna, Italy); AO Balode (Rīga Stradiṇš University, Latvia); J Miciuleviciene, R Valentiliene (Vilnius City Clinical Hospital, Lithuania); M Perrin, P Reichert, C Olinger (Laboratoire National de Sante, Luxembourg); SC de Greeff (National Institute for Public Health and the Environment (RIVM), the Netherlands); $\mathrm{H}$ Pituch, $\mathrm{P}$ ObuchWoszczatynski (Medical University of Warsaw, Poland); M Oleastro, J Machado (Instituto Nacional de Saude Dr. Ricardo Jorge, Portugal); D Lemeni, I Macovei ('Cantacuzino' National Institute of Research and Development for Microbiology and Immunology, Romania); E Nováková (Jessenius Faculty of Medicine in Martin, Slovakia); M Rupnik (National Laboratory for Health, Environment and Food, Slovenia), J Kolman (National Institute of Public Health, Slovenia); E Bouza (Hospital General Universitario ‘Gregorio Marañón', Spain), S Valdezate (Instituto de Salud Carlos III, Spain); T Åkerlund, O Aspevall (Public Health Agency of Sweden, Sweden); E Sheridan (Health Protection Agency, UK); P Rooney (Belfast City Hospital, UK); C Wiuff (Health Protection Scotland, UK); J Coia (Scottish Microbiology Reference Laboratories, UK); A Widmer (University Hospital Basel, Switzerland); M Ritzler (Labor RISCH, Liechtenstein); A Ingebretsen (Oslo University Hospital, Norway); J Bjørnholt (Norwegian Institute of Public Health, Norway); M Kroknes (St. Olavs Hospital, Norway); H Hardarson (Landspitali University Hospital, Iceland); B Levent (Refik Saydam National Hygiene Center, Turkey); M Jovanović (Clinical Center of Serbia, Serbia), M Drakulovic (Institute of Public Health of Serbia 'Dr Milan Jovanović Batut', Serbia).

\section{Acknowledgements}

This study was initiated and funded by the European Centre for Disease Prevention and Control (ECDC) through a framework service contract $(E C D C / 10 / 022)$ to Leiden University Medical Centre, Leiden, the Netherlands, for supporting capacity building for surveillance of Clostridium difficile infections at European level following an open call for tender (0J/2010/07/09-PROC/2010/035). Switzerland did not receive funding from ECDC, but contributed to the project using its own resources. The project adopted the name 'European C. difficile Infection Surveillance Network' and the acronym ECDIS-Net. The study was performed in collaboration with 
the European Study Group on C. difficile (ESGCD). The authors would like to thank Marjolein P Hensgens for advice regarding data analyses.

\section{Conflict of interest}

Sofie M van Dorp, Daan W Notermans, Jeroen Alblas, Petra Gastmeier, Katiusha Ivanova, Fidelma Fitzpatrick, Trefor Morris, Pete Kinross, Carl Suetens: none declared.

Silja Mentula: received funding and travel fees for participating in the EUCLID project from Astellas Pharmaceuticals Europe. Elisabeth Nagy: received fees for attending meetings for the EUCLID project from Astellas Pharmaceuticals Europe. Patrizia Spigaglia: received funding for participating in the EUCLID project from Astellas Pharmaceuticals Europe. Frédéric Barbut: eeceived personal fees, and non-financial support (travel expenses) from Astellas Pharma Europe, Sanofi Pasteur, Pfizer, and Merck; scientific grants from Astellas Pharma Europe, bioMérieux, bioSynex, Cepheid, Cubist, Diasorin, Quidel-Buhlmann and R-bioPharm. Mark H Wilcox: received consulting fees from Abbott Laboratories, Actelion, Astellas, Astra-Zeneca, Bayer, Cerexa, Cubist, Durata, The European Tissue Symposium, The Medicines Company, Medlmmune, Merck, Motif Biosciences, Nabriva, Optimer, Paratek, Pfizer, Roche, Sanofi-Pasteur, Seres, Summit and Synthetic Biologics; lecture fees from Abbott, Alere, Astellas, Astra-Zeneca and Pfizer; and grant support from Abbott, Actelion, Astellas, bioMerieux, Cubist, Da Volterra, The European Tissue Symposium, Merck and Summit. Ed J Kuijper: participated in advisory forums of Actelion, Astellas, Merck, Pfizer, Sanofi-Pasteur, Seres and Summit, and received unrestricted grant supports from Actelion, Merck, Becton-Dickenson and Cubist.

\section{Authors' contributions}

EK and MW were leaders of the ECDIS-Net project. DN was the leader of this project and coordinated data collection in 2011. SD coordinated data collection in 2014, analysed the data, and wrote the manuscript together with DN, EK and PK. Questionnaires were developed by DN, PK, CS, SD, FB and EK. JA adapted the web-system for data collection. All co-authors were participants in the project consortium, contributed to data collection and reviewed the manuscript.

\section{References}

1. Kuijper EJ, Coignard B, Tüll P, ESCMID Study Group for Clostridium difficile, EU Member States, European Centre for Disease Prevention and Control. Emergence of Clostridium difficile-associated disease in North America and Europe.Clin Microbiol Infect. 2006;12(Suppl 6):2-18. DOI: 10.1111/j.14690691.2006.01580.x PMID: 16965399

2. European Centre for Disease Prevention and Control (ECDC). Point prevalence survey of healthcare-associated infections and antimicrobial use in European acute care hospitals. 20112012. Stockholm: ECDC; 2013. Available from: http://www. ecdc.europa.eu/en/publications/Publications/healthcareassociated-infections-antimicrobial-use-PPS.pdf

3. Davies KA, Longshaw CM, Davis GL, Bouza E, Barbut F, Barna $Z$, et al. Underdiagnosis of Clostridium difficile across Europe: the European, multicentre, prospective, biannual, point-prevalence study of Clostridium difficile infection in hospitalised patients with diarrhoea (EUCLID). Lancet Infect Dis. 2014;14(12):1208-19. DOI: 10.1016/S1473-3099(14)70991-0 PMID: 25455988

4. Alcalá L, Martín A, Marín M, Sánchez-Somolinos M, Catalán P, Peláez T, et al. The undiagnosed cases of Clostridium difficile infection in a whole nation: where is the problem? Clin Microbiol Infect. 2012;18(7):E204-13. DOI: 10.1111/j.14690691.2012.03883.x PMID: 22563775

5. Wilcox MH. Overcoming barriers to effective recognition and diagnosis of Clostridium difficile infection.Clin Microbiol
Infect. 2012;18(Suppl 6):13-20. DOI: 10.1111/1469-0691.12057 PMID: 23121550

6. Crobach MJ, Dekkers OM, Wilcox MH, Kuijper EJ. European Society of Clinical Microbiology and Infectious Diseases (ESCMID): data review and recommendations for diagnosing Clostridium difficile-infection (CDI).Clin Microbiol Infect. 2009;15(12):1053-66. DOI: 10.1111/j.1469-0691.2009.03098.x PMID: 19929972

7. Planche T, Wilcox M. Reference assays for Clostridium difficile infection: one or two gold standards?] Clin Pathol. 2011;64(1):1 5. DOI: $10.1136 /$ jcp.2010.080135 PMID: 21118850

8. Carroll KC. Tests for the diagnosis of Clostridium difficile infection: the next generation. Anaerobe. 2011;17(4):170-4. DOI: 10.1016/j.anaerobe.2011.01.002 PMID: 21376826

9. Shetty N, Wren MW, Coen PG. The role of glutamate dehydrogenase for the detection of Clostridium difficile in faecal samples: a meta-analysis.J Hosp Infect. 2011;77(1):1-6. DOI: 10.1016/j.jhin.2010.07.024 PMID: 21145132

10. O'Horo JC, Jones A, Sternke M, Harper C, Safdar N. Molecular techniques for diagnosis of Clostridium difficile infection: systematic review and meta-analysis. Mayo Clin Proc. 2012 Jul;87(7):643-51. DOl: http://dx.doi.org/10.1016\%2Fj. mayocp.2012.02.024 PMID: 22766084

11. Crobach MJ, Planche T, Eckert C, Barbut F, Terveer EM, Dekkers $O M$, et al. European Society of Clinical Microbiology and Infectious Diseases: update of the diagnostic guidance document for Clostridium difficile infection. Clin Microbiol Infect. 2016. Forthcoming.

12. Lewis SJ, Heaton KW. Stool form scale as a useful guide to intestinal transit time.Scand J Gastroenterol. 1997;32(9):920-4. DOI: 10.3109/00365529709011203 PMID: 9299672

13. Bartlett JG, Gerding DN. Clinical recognition and diagnosis of Clostridium difficile infection.Clin Infect Dis. 2008;46(s1) Suppl 1;S12-8. DOI: 10.1086/521863 PMID: 18177217

14. Barbut F, Surgers L, Eckert C, Visseaux B, Cuingnet $M$, Mesquita $C$, et al. Does a rapid diagnosis of Clostridium difficile infection impact on quality of patient management? Clin Microbiol Infect. 2014;20(2):136-44. DOI: 10.1111/14690691.12221 PMID: 23565919

15. Longtin Y, Trottier S, Brochu G, Paquet-Bolduc B, Garenc C, Loungnarath $\mathrm{V}$, et al. Impact of the type of diagnostic assay on Clostridium difficile infection and complication rates in a mandatory reporting program. Clin Infect Dis. 2013;56(1):67-73. DOI: $10.1093 / \mathrm{cid} / \mathrm{cis} 840$ PMID: 23011147

16. Huber CA, Foster NF, Riley TV, Paterson DL. Challenges for standardization of Clostridium difficile typing methods. J Clin Microbiol 2013 Sep;51(9):2810-4. DOI: http://dx.doi. org/10.1128\%2FJCM.00143-13 PMID: 23784128

17. Fawley WN, Knetsch CW, MacCannell DR, Harmanus C, Du $\mathrm{T}$, Mulvey MR, et al. Development and validation of an internationally-standardized, high-resolution capillary gelbased electrophoresis PCR-ribotyping protocol for Clostridium difficile. PLoS One. 2015;10(2):e0118150. DOI: 10.1371/journal. pone.0118150 PMID: 25679978

18. Knetsch CW, Lawley TD, Hensgens MP, Corver J, Wilcox MW, Kuijper EJ. Current application and future perspectives of molecular typing methods to study Clostridium difficile infections. Euro Surveill. 2013;18(4):20381.PMID: 23369393

19. He M, Miyajima F, Roberts P, Ellison L, Pickard DJ, Martin MJ, et al. Emergence and global spread of epidemic healthcareassociated Clostridium difficile. Nat Genet 2013 Jan;45(1):10913. DOI: http://dx.doi.org/10.1038\%2Fng.2478 PMID: 23222960

20. Bauer MP, Notermans DW, van Benthem BH, Brazier JS, Wilcox $\mathrm{MH}$, Rupnik $\mathrm{M}$, et al. Clostridium difficile infection in Europe: a hospital-based survey. Lancet. 2011;377(9759):63-73. DOI: 10.1016/S0140-6736(10)61266-4 PMID: 21084111

21. European Clostridium difficile Surveillance Network (ECDISNet). Protocols and documents. Berlin: ECDIS-Net. [Accessed 16 May 2016]. Available from: http://ecdisnet.eu/index.php/ home/protocol

22. Rossi E, Vita A, Baccetti S, Di Stefano M, Voller F, Zanobini A. Complementary and alternative medicine for cancer patients: results of the EPAAC survey on integrative oncology centres in Europe.Support Care Cancer. 2015;23(6):1795-806. DOI: 10.1007/S00520-014-2517-4 PMID: 25471177

23. Planche TD, Davies KA, Coen PG, Finney JM, Monahan IM, Morris KA, et al. Differences in outcome according to Clostridium difficile testing method: a prospective multicentre diagnostic validation study of $C$ difficile infection. Lancet Infect Dis. 2013;13(11):936-45. DOI: 10.1016/S1473-3099(13)70200-7 PMID: 24007915

24. Bowker AH. A test for symmetry in contingency tables.J Am Stat Assoc. 1948;43(244):572-4. DOI: 10.1080/01621459.1948.10483284 PMID: 18123073 
25. The Clostridium difficile subcommittee of the Scientific Advisory Committee of the Health Protection Surveillance Centre (HPSC) Surveillance, diagnosis and management of Clostridium difficile infection in Ireland. National Clinical Guideline No. 3. Dublin: Department of Health; 2014. Available from: http://health.gov.ie/wp-content/uploads/2015/01/ National-Clinical-Guideline-No.-3-Clostridium-difficile.pdf

26. $\mathrm{ADH} / \mathrm{HCAl} /$ infectious disease. Updated guidance on the diagnosis and reporting of Clostridium difficile. London: Department of Health; 2012. Available from: https://www. gov.uk/government/publications/updated-guidance-on-thediagnosis-and-reporting-of-clostridium-difficile

27. Barbut F, Delmée M, Brazier JS, Petit JC, Poxton IR, Rupnik M, et al. A European survey of diagnostic methods and testing protocols for Clostridium difficile. Clin Microbiol Infect. 2003;9(10):989-96. DOI: 10.1046/j.1469-0691.2003.00683.x PMID: 14616740

28. Gould CV, Edwards JR, Cohen J, Bamberg WM, Clark LA, Farley $M M$, et al. Effect of nucleic acid amplification testing on population-based incidence rates of Clostridium difficile infection. Clin Infect Dis. 2013;57(9):1304-7. DOI: 10.1093/cid/ cit492 PMID: 23899677

29. Wilcox MH, Shetty N, Fawley WN, Shemko M, Coen P, Birtles $A$, et al. Changing epidemiology of Clostridium difficile infection following the introduction of a national ribotypingbased surveillance scheme in England. Clin Infect Dis. 2012;55(8):1056-63. DOI: 10.1093/cid/cis614 PMID: 22784871

30. Alcalá L, Marín M, Martín A, Sánchez-Somolinos M, Catalán P, Peláez MT, et al. Laboratory diagnosis of Clostridium difficile infection in Spain: a population-based survey. J Hosp Infect. 2011;79(1):13-7. DOI: 10.1016/j.jhin.2011.05.017 PMID: 21741114

31. Alcalá L, Reigadas E, Marín M, Martín A, Catalán P, Bouza E, Spanish Clostridium difficile Study Group. Impact of clinica awareness and diagnostic tests on the underdiagnosis of Clostridium difficile infection. Eur J Clin Microbiol Infect Dis. 2015;34(8):1515-25. DOI: 10.1007/s10096-015-2380-3 PMID: 25904126

32. Bouza E, Marín M, Peláez T, Alcalá L, Group for Clostridium difficile Infection of the Spanish Society for Chemotherapy. The situation and management of Clostridium difficile infection in Spain: an opinion document.Rev Esp Quimioter. 2013;26(3):261-86.PMID: 24080894

33. Pituch H, Obuch-Woszczatyński P, Lachowicz D, Wultańska D, Karpiński P, Młynarczyk G, et al. Hospital-based Clostridium difficile infection surveillance reveals high proportions of PCR ribotypes 027 and 176 in different areas of Poland, 2011 to 2013. Euro Surveill. 2015;20(38):30025. DOI: 10.2807/15607917.ES.2015.20.38.30025 PMID: 26536049

34. European Centre for Disease Prevention and Control (ECDC). Minutes of the 39th meeting of the Advisory Forum, Stockholm, 24-25 September 2014. Stockholm: ECDC; 2014. Available from: http://ecdc.europa.eu/en/aboutus/governance/af/ AF\%20\%20Meeting\%20Minutes/Advisory-Forum-minutesmeeting-39.pdf

\section{License and copyright}

This is an open-access article distributed under the terms of the Creative Commons Attribution (CC BY 4.0) Licence. You may share and adapt the material, but must give appropriate credit to the source, provide a link to the licence, and indicate if changes were made.

This article is copyright of the authors, 2016. 